\title{
Calculation of Distributed Photovoltaic Grid Acceptance Capacity Considering Timing Characteristics of Photovoltaic Output
}

\author{
Xue WANG ${ }^{1, \mathrm{a},{ }^{*}, \text { Qiang SUN }}{ }^{1, \mathrm{~b}}$, Ying-ting NI ${ }^{2, \mathrm{c}}$ and Qiang YIN $^{2, \mathrm{~d}}$ \\ ${ }^{1}$ State Grid Energy Research Institute, Beijing 102209, China; \\ ${ }^{2}$ Key Laboratory of Smart Grid of Ministry of Education, Tianjin University, Tianjin 300072,China; \\ email: ${ }^{a}$ wangxue@sgeri.sgcc.com.cn, ${ }^{b}$ sunqiang@sgeri.sgcc.com.cn, ${ }^{c}$ luofengzhang@tju.edu.cn
}

\begin{abstract}
The smart power network is development trend of the modern power system, which is an important carrier and composition part for current energy source revolution in the world. Construction of the smart power network brings profound influence on the traditional power distribution network. Development mode, structure function, operation characteristic and comprehensive benefit etc aspects of the smart power distribution network will change obviously, the double flows caused by access of the distribution type photovoltaic is one of the important change of the smart power distribution network. Access way and access scale of the distribution type photovoltaic will directly affect planning and construction of the power distribution network in future, and output time series characteristic of photovoltaic also increases complication degree of photovoltaic acceptance capacity calculation. This paper establishes the optimization model of the photovoltaic acceptance capacity counting output time series characteristic of photovoltaic, which studies maximum acceptance capacity of the distribution type photovoltaic considering stable restrain. The sample shows the analysis method forwarded in this paper is applicable to maximum acceptance capacity analysis of photovoltaic meeting with stable restrain of the system.
\end{abstract}

Keywords-photovoltaic output; time series characteristic; distribution type photovoltaic; acceptance capacity

\section{FOREWORD}

In recent year, with the rapid development of the photovoltaic industry and all-round construction of the smart power distribution network, capacity acceptance issue of the photovoltaic system becomes the hot spot research subject. Acceptance capacity of photovoltaic is defined as proportion between maximum access capacity of photovoltaic power generation and maximum load of the line. Domestic and foreign study about synchronization acceptance capacity of the renewable energy sources power generation system mostly focus on research of wind power[1][2][3]; and study on acceptance capacity of the photovoltaic synchronization lays special emphasis on research of the large scale photovoltaic power station etc centralization photovoltaic power generation system with installation capacity of 6MW and above, study on the distribution type photovoltaic in the power distribution network is rare.
In recent year, some scholars have carried out analysis and research on factors which affect acceptance capacity of the distribution type photovoltaic from theoretical perspective. The document [4] analyzes fluctuation of the distribution type photovoltaic access on voltage and current of the system, which finds out limit positions for voltage and current over limit, and the relationship expression between the maximum acceptance capacity and the node current, line current is found out through curve fitting. But the fitting method is only applicable to special algorithm case. It isn't applicable to the more photovoltaic access point and complicate network case. The document [5] also analyzes influence of voltage fluctuation, load and power factor etc of the high level substation on photovoltaic acceptance capacity. The document [6] takes voltage stability, power factory and transient harmonic etc of the system as restrain conditions, which carries out calculation and analysis of maximum access capacity of photovoltaic. The document [7] takes voltage deviation, voltage fluctuation etc general energy quality index as restrain conditions, which analyzes maximum acceptance capacity of photovoltaic at different power factor for power generation. But these researches mostly focus on the single point access system of photovoltaic. Because system flow under the multiple power source point condition is different to that under single point access, it shall be considered independently, and study isn't related to influence of time series characteristic of photovoltaic on maximum access capacity of photovoltaic.

\section{STUDY OF DISTRIBUTION TYPE PHOTOVOLTAIC ACCEPTANCE CAPACITY BASED ON STABLE RESTRAINT}

Studying maximum acceptance capacity of the distribution type photovoltaic is actually to solve the maximum access capacity issue of the distribution type photovoltaic under restrain condition of the system. Access efficiency of the distribution type photovoltaic is determined by access capacity and installation capacity of photovoltaic. Therefore solving maximum access capacity issue of the distribution type photovoltaic is study on maximum acceptance capacity of photovoltaic on basis of considering efficiency. Generally speaking, higher is load of the system, greater is the access capacity of photovoltaic. It shall start 
from maximum load of the system when maximum acceptance capacity of the distribution type photovoltaic is solved, various stable restrain condition of the system shall be considered and the photovoltaic access capacity shall be optimized. Considering maximum limit condition of load, the method forwarded in this paper finds out stability analysis of the maximum acceptance capacity of the distribution type photovoltaic on the acceptance capacity at every position assuming the standby position is determined, and action of the voltage distortion and harmonic influence are neglected. The maximum access capacity of photovoltaic is taken as the objective function, the system flow, node voltage and thermal stability (line power) etc are taken as restrain conditions.

$$
\max f(X)=\sum_{i=1}^{n} P_{P V, i}(\mathrm{X})
$$

In which, $P_{P V, i}$ is installation capacity of No i distribution type photovoltaic system; $n$ is number of access point of photovoltaic; optimization variable $\mathrm{X}$ is configuration condition of the distribution type photovoltaic, which is $\mathrm{n}$ dimension vector; now we only discuss the case that power factor for photovoltaic power generation $\mathrm{PF}=1$, i.e., the photovoltaic power supply only has active power output. line.

Equality restrain is: the power balance equation of the

$$
\left\{\begin{array}{l}
P_{P V i}-P_{L i}-U_{i} \sum_{j=1}^{N} U_{j}\left(G_{i j} \cos \delta_{i j}+B_{i j} \sin \delta_{i j}\right)=0 \\
Q_{P V i}-Q_{L i}-U_{i} \sum_{j=1}^{N} U_{j}\left(G_{i j} \sin \delta_{i j}-B_{i j} \cos \delta_{i j}\right)=0
\end{array}\right.
$$

In which, $P_{P V i}$ and $Q_{P V i}$ are active power and inactive power of no $i$ point distribution type photovoltaic respectively; $P_{L i}$ and $Q_{L i}$ are active power and inactive power of no $i$ point respectively; $U_{i}$ and $U_{j}$ are node voltage of node $i$ and $\mathrm{j} ; \delta_{i j}$ is phase angle difference, $G_{i j}$ and $B_{i j}$ are admittances of the system.

Inequality restrain including:

(1) Voltage restrain of the node:

$$
U_{i \min } \leq U_{i} \leq U_{i \max }
$$

In which, $U_{i}$ is node voltage of node $i ; U_{i \max }$ and $U_{i \min }$ are upper limit and lower limit of voltage of node $i$.

(2) Thermal stability restrain of line:

$$
S_{\text {line }, i} \leq S_{\text {line }, i \max } \quad(i=1,2, \cdots, N l)
$$

In which, $N l$ is total number of the line; $S_{\text {line }, i}$ and $S_{\text {line, } i \max }$ are power of No $i$ branch and upper limit of allowable power of the line.

(3) Installation capacity restrain of single unit of photovoltaic;

$$
S_{p v, i \min } \leq S_{i} \leq S_{p v, i \max } \quad(i=1,2, \cdots, n)
$$

In which, $S_{i}$ is apparent power no $i$ distribution type photovoltaic; $S_{p v, \text { min }}$ and $S_{p v, i \max }$ are lower limit and upper limit of apparent power of no $i$ photovoltaic, $n$ is total point number of the access photovoltaic.

(4) Total capacity restrain of photovoltaic:

$$
\sum_{i=1}^{n} P_{i} \leq \alpha \sum_{j=1}^{N} P_{\text {load }}
$$

In which, $P_{i}$ is active power of no $i$ unit distribution type photovoltaic; $\sum_{j=1}^{N} P_{\text {load }}$ is total load of the power distribution grid; $\alpha$ is proportion coefficient of allowable photovoltaic access. Total capacity of the distribution type power supply shall not exceed $25 \%$ maximum load within power supply area of the upper level transformer in principle according to regulations of enterprise standard Q/GDW480 - 2010 of State Grid Corporation about access grid technology of the distribution type power supply, $\alpha$ generally takes $25 \%$. Because this paper carries out theoretical analysis on acceptance capacity, requirement is suitably widened. Capacity of access photovoltaic is required to be lower than total load of the area $(\alpha=1)$, power reverse phenomenon is not allowed.

\section{TIME SERIES CALIBRATION METHOD}

Learn from analysis of system load and own time series characteristic of photovoltaic, peak period of load and peak value of photovoltaic output are always not located within same time section, and plus intermittence and fluctuation of photovoltaic generated by lighting, the photovoltaic configuration plan which is solved to contain maximum capacity still can make a part of restrain conditions over limit during actual operation[8]. Therefore this configuration plan shall be valid in every time section within operation period, and carry out flow calculation of every time section and observe over limit condition of node voltage and line power of every section.

In case node voltage or thermal stability restrain at some moment exceeds limit, it means access capacity of photovoltaic at this moment exceeds allowable value, and maximum capacity of photovoltaic shall be limited. Initial value of the limit coefficient $\alpha$ can take $90 \%$, if node voltage or thermal stability restrain still exceeds limit when 
access capacity of photovoltaic is within limit scope, continue reduce the limit coefficient until it doesn't exceed limit, Step length $\beta$ takes $10 \%$. If every restrain condition doesn't exceed restrain under some one photovoltaic configuration capacity, start to increase the limit coefficient,

the increased step length $\beta$ can be $5 \%$, and start to calculate over limit condition again. If it doesn't exceed limit, continue increase the limit coefficient, the increased step length $\beta$ is changed to $2.5 \%$; if it exceeds limit, and then reduce the limit coefficient, the reduced step length $\beta$ is $2.5 \%$, and carry out iteration on basis of it. Change quantity $\beta$ of limit coefficient for every iteration is 0.5 times last coefficient until $\beta$ reaches some value (it takes $1.25 \%$ in this paper).

The photovoltaic configuration plan which is finally determined will unlimitedly trend to maximum access capacity of photovoltaic required by every restrain condition of time series. Furthermore for the node with high acceptance capacity of the output system (such as the node at inlet end of the feeding wire), initial value of $\alpha$ can select small one, it is beneficial to quick convergence of iteration. Flow chart for time series analysis calibration of the distribution plan of the distribution type photovoltaic under operation restrain is shown as fig. 1.

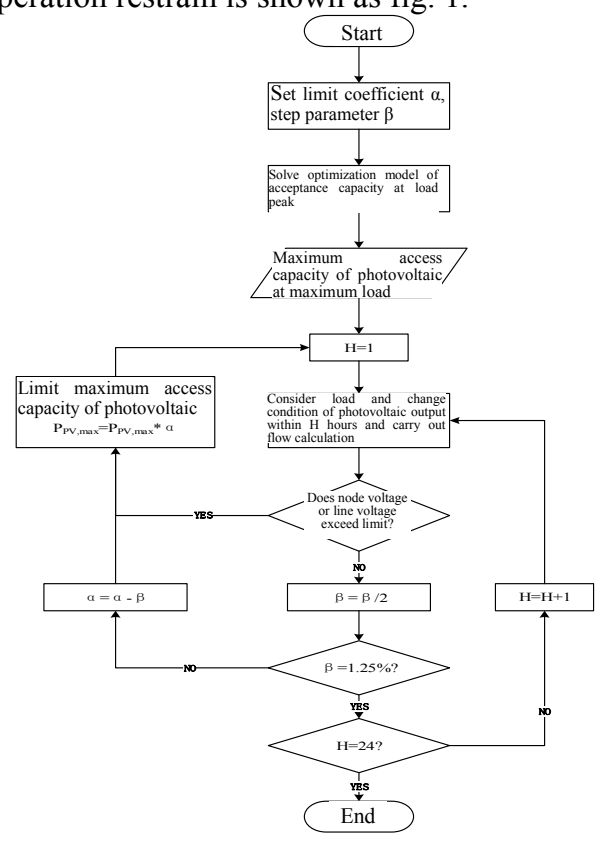

Figure 1. Flowchart of timing verification

\section{Cuckoo SEARch Algorithm}

Cuckoo Search Algorithm (Cuckoo Search Algorithm, abbreviated as CSA) is a new method which is all round quick search of population iteration simulating nest searching and hatch behavior of Cuckoo. The Cuckoo bird has a very special reproductive behavior, its hatch reproduction of later generation is completed through laying bird eggs in the nest of other birds (host bird). Through select the bird nest with good quality and improve the nest with bad quality, the Cuckoo finally finds out the nest with best quality to hatch. Under general conditions, the following 3 ideal status are usually set to simulate nest searching behavior of the Cuckoo[9]:

(1) The Cuckoo only lay one egg one time, the nest position is randomly select to hatch;

(2) In a group of the nests which are randomly selected, the nest with good quality is remained for next generation;

(3) Quantity n of the nest which can be used is fixed, possibility that the host bird finds the egg of the Cuckoo bird is $D_{\mathrm{a}} \in[0,1]$.

Furthermore, the Cuckoo applies Levy (Levy) fly mode which refers to the current found nest position with optimum quality during optimization searching process, which forms a high efficient all round optimization searching mode.

Therefore, CSA is characterized with simple concept, less adjustment parameters, quick optimization searching speed, high search accuracy and not liable to be trapped in local optimization etc advantages. Its concrete optimization searching steps are shown as following:

Step 1: Initialization of parameters of the Cuckoo search algorithm. Number of the nest which is generated by initialization is N, possibility that the Cuckoo egg is found by the host bird is $D_{a}$;

Step 2: Initializing position of the nest.Positions of $\mathrm{N}$ nests $X_{i}=\left[X_{1}, X_{2}, \ldots, X_{m}\right], \quad i=1,2, \ldots N \pi$ are randomly generated;

Step 3: evaluate quality of the nest. Determine the objective function, i.e., quality of the nest in this algorithm, and record position of the nest with optimum quality $X_{i}=\left[X_{1}, X_{2}, \ldots, X_{m}\right]$ and its best quality bestf ;

Step 4: Position of the nest is improved by Levy fly mode. Positions of $\mathrm{N}$ nests are improved as formula (7):

$$
X_{i}^{t+1}=X_{i}^{t}+\alpha \oplus L
$$

In which, $\mathrm{t}$ is current iteration times; $\oplus$ means point to point multiply; $\alpha$ is control parameter of the step length, its value follows standard positive distribution; L is Levy search route, i.e., the step length during flying:

$$
L=0.01 \times \frac{u}{|v|^{1 / \beta}}\left(X_{\text {best }}-X_{i}^{t}\right)
$$

In which, coefficient 0.01 is typical fly size in Levy fly mode; $\beta=3 / 2 ; X_{\text {best }}$ means the current nest with best 
quality; $u$ and $v$ are random number of normal distribution respectively.

$$
u \sim N\left(0, \sigma_{u}^{2}\right), v \sim N\left(0, \sigma_{v}^{2}\right)
$$

$$
\text { In which, } \sigma_{u}=\left[\frac{\Gamma(1+\beta) \sin (\pi \beta / 2)}{2^{(1+\beta) / 2} \Gamma((1+\beta) / 2) \beta}\right]^{-1 / \beta} \text {, }
$$$$
\sigma_{v}=1
$$

In which, $\Gamma$ is standard gamma function.

Step 5: replace position of the nest. Calculate quality of every nest after improvement, compare quality of every nest before improvement, and maintain $\mathrm{N}$ nests with best quality according to greed law;

Step 6: improve position of the nest according to find possibility $D_{a}$. For $\mathrm{N}$ nests, $r_{i} \in[0,1]$ is randomly taken. If $r_{i} \leq D_{a}$ and it isn't found, position of the nest isn't improved; in case $r_{i}>D_{a}$, it is found, and position improvement of the nest is carried out according to formula (10):

$$
X_{i}^{(t+1)}=X_{i}^{(t)}+r_{i}\left(X_{j}^{(t)}-X_{i}^{(t)}\right)
$$

In which, $X_{j}^{(t)}$ is a nest near $X_{i}^{(t)}$.

Step 7: replace position of the nest. Calculate quality of every nest after improvement, and compare quality of every nest before improvement, and $\mathrm{N}$ nests with good quality are maintained according to greed law;

Step 8: record position and quality of the optimum nest. Record the nest position $X_{\text {best }}$ with optimum quality and optimum quality bestf during this iteration;

Step 9: Judge whether it meets with termination condition. If it meets with termination condition, iteration is stopped, the optimum nest quality bestf is outputted; otherwise it is transferred to step 4 and carry out optimization search again.

\section{ALGORITHM ANALYSIS}

The algorithm in this paper applies the IEEE123 node algorithm, it is a constant power load mode. Both load node and photovoltaic are considered as PQ nodes. Total active power of the system is $3.48379 \mathrm{MW}$, total inactive power is 1.35884Mvar. Reference value of three phase power $\mathrm{S}_{\mathrm{B}}=5000 \mathrm{kVA}$, and reference value of line voltage $\mathrm{U}_{\mathrm{B}}=4.16 \mathrm{kV}$.

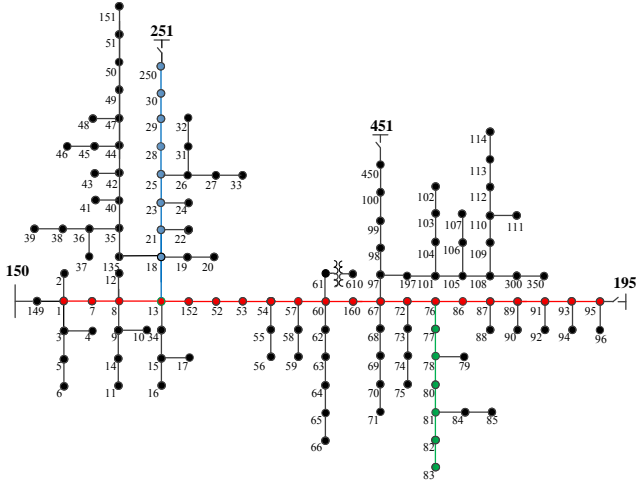

Figure 2. IEEE 123 nodes test case

(1) Single point access condition of photovoltaic

Select the main trunk line in the algorithm: 1-7-8-13152-52-53-54-57-60-160-67-72-76-86-87-89-91-93-95 and two branch lines: the branch line 1 is 18-21-23-25-28-29$30-250$, the branch line 2 is 77-78-80-81-82-83, and calculate maximum acceptance capacity of every node respectively. Pre-unit value of the node voltage is controlled within $[0.95,1.05]$. Calculation result is shown as fig. 3 .

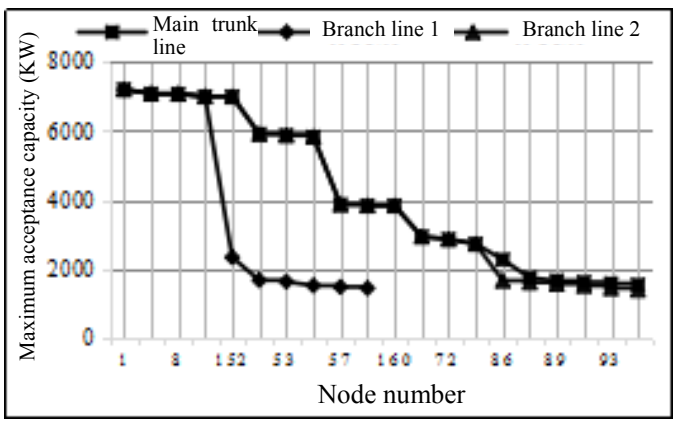

Figure 3. Maximum acceptable capacity of PV nodes

Seen from the whole trend of the curve, the farther the synchronization point of the distribution type photovoltaic to the outlet of the feed line is, the smaller the maximum acceptance capacity of the node is. The acceptance capacity of the distribution type photovoltaic of the node at the inlet of the feed line is strongest. Because influence of the distribution type photovoltaic accessed at inlet of the feed line on voltage amplitude of every node. When photovoltaic capacity is increased, rise amplitude of voltage at every node is smaller than the case that the distribution type photovoltaic is accessed at end of the feed line, it will reach voltage restrain and restrain limit of power of the line.

Seen from fig. 3, the photovoltaic acceptance capacity of the main trunk line is higher than the node on the corresponding branch line. After the main trunk line is furcated from the branch line, acceptance capacity of the first node (No 18 and No 77 node) on the branch line drops down obviously. Following extension of the branch line, change amplitude of the acceptance capacity isn't great. Generation of photovoltaic acceptance capacity difference between the nodes on the main trunk line and the branch 
line is caused by more connection points on the main trunk line and every branch line. When power of the line rises up, the main trunk line can transfer power through its connected node.

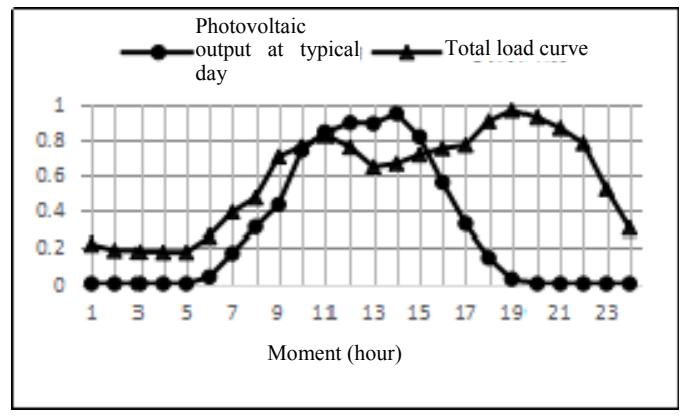

Figure 4. Typical PV output in typical day

The main trunk line in the algorithm: 1-7-8-13-152-52$53-54-57-60-160-67-72-76-8$

6-87-89-91-93-95 is selected as the optional point for photovoltaic access. Validation result of analysis which considers maximum photovoltaic acceptance capacity of every node during time series validation is shown as fig. 5 .

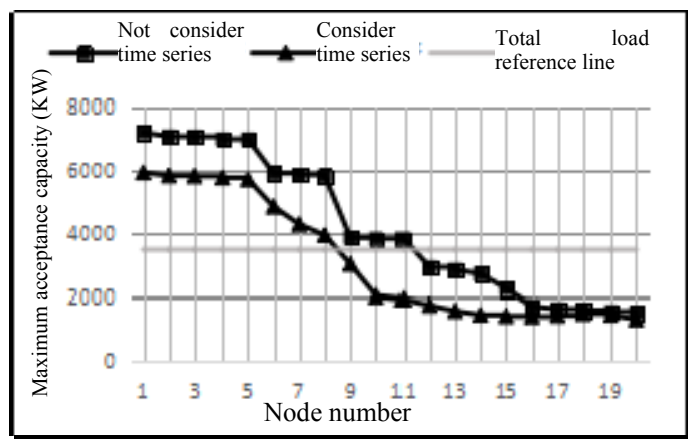

Figure 5. Maximum acceptable capacity of PV considering time-series analysis method

Seen from fig. 5, maximum photovoltaic acceptance capacity of every node on the main trunk line drops down during time series validation, acceptance capacity at the outlet section of the line is close to condition no time series validation. Furthermore, the node at the inlet of the feed line is mostly limited by power restrain of the line, current of the line reaches limit value earlier than voltage. Situation of the node at outlet is reverse, it will be firstly limited by voltage of the node. Because sensitivity of node voltage at the inlet of the line to photovoltaic access is very low, and change is slow.

Most over limit conditions occur after 12 hours or 13 hours. The load curve has obvious drop trend within this time duration, but photovoltaic output rises up continuously, shown as figure 5, which causes a part of output not be locally consumed, voltage of other nodes on the line rises up and maximum acceptance capacity of photovoltaic is limited.

Total active power load of the system is $3483.79 \mathrm{~kW}$. When multiple points access of photovoltaic is considered, the maximum acceptance capacity of photovoltaic shall be limited, and power reverse isn't allowed, and then set $\sum P_{P V} \leq 3490$. Take the comprehensive load curve as a sample and analyze acceptance capacity of multiple points access. Penetration rate of photovoltaic is defined as proportion between acceptance capacity of photovoltaic and maximum load.

(2)Multiple point access case of photovoltaic

Firstly select the line 160-67-72-76-86-87-89-91-93-95 on the main trunk line and two branch circuits 23-25-28-29$30,77-78-80-81-82$ and optional access point of photovoltaic, and analyze acceptance capacity of photovoltaic.

When time series validation isn't considered, the optimized photovoltaic maximum acceptance capacity is $3490 \mathrm{~kW}$, which reaches the specified limit, i.e., total active power.

When maximum photovoltaic acceptance capacity of every node is validated within 24 hours in typical day, optimization result of maximum acceptance capacity is shown as table 1.

TABLE 1. INSTALLATION CAPACITY OF DISTRIBUTED PV FOR EVERY NODE IN LINE

\begin{tabular}{|c|l|l|l|l|l|}
\hline $\begin{array}{c}\text { Node (main } \\
\text { trunk line) }\end{array}$ & 160 & 67 & 72 & 76 & 86 \\
\hline $\begin{array}{c}\text { PV } \\
\text { capacity/kW }\end{array}$ & 30 & 110 & 120 & 110 & 270 \\
\hline $\begin{array}{c}\text { Node (main } \\
\text { trunk line) }\end{array}$ & 87 & 89 & 91 & 93 & 95 \\
\hline $\begin{array}{c}\text { PV capacity } \\
\text { /kW }\end{array}$ & 140 & 130 & 30 & 190 & 30 \\
\hline $\begin{array}{c}\text { Node (main } \\
\text { trunk line) }\end{array}$ & 23 & 25 & 28 & 29 & 30 \\
\hline $\begin{array}{c}\text { PV capacity } \\
\text { /kW }\end{array}$ & 180 & 30 & 80 & 240 & 50 \\
\hline $\begin{array}{c}\text { Node } \\
\text { (branch line) }\end{array}$ & 47 & 48 & 49 & 50 & 51 \\
\hline $\begin{array}{c}\text { PV capacity } \\
/ \mathrm{kW}\end{array}$ & 210 & 60 & 150 & 170 & 80 \\
\hline $\begin{array}{c}\text { Node } \\
\text { (branch line) }\end{array}$ & 77 & 78 & 80 & 81 & 82 \\
\hline $\begin{array}{c}\text { PV capacity } \\
/ \mathrm{kW}\end{array}$ & 110 & 200 & 180 & 80 & 30 \\
\hline
\end{tabular}

The optimized photovoltaic acceptance capacity is $3280 \mathrm{~kW}$, In photovoltaic penetration rate by timing series verification, the maximum acceptance capacity of photovoltaic is reduced.

Furthermore, for a part of the point with high single point acceptance capacity, such as 160 , its installation capacity will not be very high when total acceptance capacity for multiple point access is maximum. Because it isn't same as the single point access case, the node will be affected by photovoltaic access of other near nodes. Access of several distribution type photovoltaic will generate superposition effect on voltage rise near the access point, which will generate obvious rise function. 
The partial nodes in above lines are selected as the optional node for photovoltaic access and carry out analysis.

TABLE2. INSTALLATION CAPACITY OF DISTRIBUTED PV FOR PARTIAL NODES

\begin{tabular}{|c|c|c|c|c|}
\hline Access point & $\begin{array}{c}\text { Capacity of } \\
\text { access } \\
\text { photovoltaic } \\
\text { for every } \\
\text { node }\end{array}$ & $\begin{array}{c}\text { General } \\
\text { accepta } \\
\text { nce } \\
\text { capacity }\end{array}$ & $\begin{array}{c}\text { photov } \\
\text { oltaic } \\
\text { penetrat } \\
\text { ion rate }\end{array}$ & $\begin{array}{c}\text { Maximum } \\
\text { acceptance } \\
\text { capacity of } \\
\text { single point }\end{array}$ \\
\hline $\begin{array}{c}23-25-28-29- \\
30\end{array}$ & $\begin{array}{c}40,130,110,2 \\
50,1100\end{array}$ & 1630 & $46.70 \%$ & 1520 \\
\hline $\begin{array}{c}23-25-28-29- \\
30,\end{array}$ & $\begin{array}{c}120,0,400,26 \\
0,270\end{array}$ & 2360 & $67.62 \%$ & 1520 \\
$57-48-49-50-$ & $\begin{array}{c}380,350,90,2 \\
30,260\end{array}$ & & & \\
\hline $\begin{array}{c}23-25-28-29- \\
30,\end{array} 7-48-49-50-$ & $\begin{array}{c}0,0,0,460,530 \\
450,0,330,21 \\
51,\end{array}$ & $0,0,140,60,31$ \\
$77-78-80-81-$ & $0,0,300$ & 2790 & $79.94 \%$ & 1610 \\
82 & & & & \\
\hline
\end{tabular}

Seen from table 2, acceptance capacity of the system on the multiple point access photovoltaic is stronger than acceptance capacity of single node in the line. When access point is more, rise amplitude is obvious; when number of the access point is less, rise amplitude isn't great. Now the acceptance capacity of the line is close to maximum acceptance capacity of single node (it is generally the node at starting end of the line). When the single point is accessed, acceptance capacity of the node at starting point is very high, the node at rear end firstly reaches voltage limit phenomenon when capacity of the accessed distribution type photovoltaic rises up, the final limit occurs on the node at starting end.

\section{CONCLUSION}

This paper establishes the optimization model of photovoltaic acceptance capacity in time series analysis frame, which studies maximum acceptance capacity issue of the distribution type photovoltaic considering stable restrain, and the Cuckoo algorithm which is applicable to solve photovoltaic acceptance capacity issue is forwarded.

Through discussion of different access condition of photovoltaic, acceptance capacity of the distribution type photovoltaic considering stable restrain is studied, the maximum photovoltaic acceptance capacity which meets with stable restrain of the system is solved, and validation of acceptance capacity solution method forwarded in this paper is verified.

\section{REFERENCES}

[1] Zhang Yong, Study of wind power accommodated capacity of power grid based on time series simulation, [D]. Southwest Jiaotong University, 2012.

[2] Ren Hua, Constraint Conditions and influence factors of wind power penetration, [D]. Beijing Jiaotong University, 2014.

[3] Ai Ziziyangapal, Capacity of power system on acceptance of intermittent energy [D]. Beijing Jiaotong University, 2012.

[4] Yuan Yuan, LiuTianqi, Cheng Daowe, Research on maximum access capacity of grid-connected photovoltaic power [J].Renewable Energy Resources, 2012, 30(6): 9-14.

[5] Rafael AmaralShayani. Photovoltaic Generation Penetration Limits in Radial Distribution Systems [J]. IEEE Transactions on Power Systems, 2011, 26(3).

[6] Ye Jingen, Zhu Mingxing, Li Lingdong, Simulation analysis on the capacity of distribution network to accept distributed photovoltaic power [J]. High Power Converter Technology, 2011, 1(3): 29-33.

[7] Li Xiaoming, Wang Kaihong, DuanXiaohong, et al, Analysis on maximum power generation of photovoltaic generation in distribution network [J]. Hebei Electric Power, 2012, 31(5): 17-19.

[8] Li Liang, Tang Wei, Bai Mu, et al, Multi-objective Locating and Sizing of Distributed Generators based on time-series characteristics [J]. Automation of Electric Power Systems, 2013, 37(3): 58-63.

[9] Chen Ling, Wang Honghua, Han Wei, Simulation study of photovoltaic power generation in maximum power point tracking based on CSA-FPI algorithm[J]. Electrical Measurement \& Instrumentation, 2015, 8: 39-44. 